\title{
Two distinct destabilizing elements in the c-fos message trigger deadenylation as a first step in rapid mRNA decay
}

\author{
Ann-Bin Shyu, ${ }^{1}$ Joel G. Belasco, and Michael E. Greenberg \\ Department of Microbiology and Molecular Genetics, Harvard Medical School, Boston, Massachusetts 02115 USA
}

The mechanisms by which c-fos mRNA is targeted for decay have been examined. Rapid removal of the poly(A) tail occurs before the transcribed portion of the c-fos message is degraded. Identification of the determinants that mediate c-fos message deadenylation reveals that they coincide directly with previously characterized determinants of c-fos mRNA instability, one in the protein-coding region and the other an AU-rich element (ARE) in the 3 '-untranslated region. Insertion of either of these c-fos instability elements into the stable $\beta$-globin message confers the property of rapid deadenylation. Mutation of the ARE indicates that this sequence controls two steps in the process of c-fos mRNA degradation: removal of the poly(A) tail, which does not require intact AUUUA pentanucleotides within the ARE, and subsequent degradation of the transcribed portion of the message, which appears to be dependent on the AUUUA pentanucleotides. These results indicate that structurally distinct instability determinants within the transcribed portion of labile messages can function by promoting rapid removal of the poly(A) tail as a first step in the decay process.

[Key Words: c-fos; mRNA decay; deadenylation; poly(A); Au-rich element]

Received September 27, 1990; revised version accepted November 26, 1990.

The degradation of mRNA is an important mechanism for controlling gene expression in prokaryotic and eukaryotic cells (for review, see Brawerman 1987; Belasco and Higgins 1988; Bernstein and Ross 1989). Rates of cytoplasmic mRNA turnover can vary widely within a single cell and are often influenced by environmental signals. Thus, the half-lives of some mammalian messages span several hours or even days, while other mRNAs decay within minutes of their initial synthesis. The biochemical pathways by which mRNAs are differentially targeted for degradation are largely unknown.

The c-fos proto-oncogene transcript is one of the least stable messages in mammalian cells /Greenberg and Ziff 1984). It encodes a transcription factor that is believed to regulate a diverse set of genes important for cell growth and differentiation (for reviews, see Curran and Franza 1988; Sheng and Greenberg 1990). Transcription of c-fos and many other members of the immediate early gene family is rapidly induced by growth factors and other extracellular stimuli and then ceases completely within 30-60 min after induction /Greenberg and Ziff 1984; Lau and Nathans 1985; Lim et al. 1987; Almendral et al. 1988). Tight control of the synthesis and degradation of c-fos mRNA appears to be critical for normal cellular

${ }^{1}$ Present address: Department of Biochemistry and Molecular Biology, The University of Texas Health Science Center, Medical School, Houston, Texas 77030 USA. function, inasmuch as deregulated expression of this gene can lead to oncogenic transformation (Miller et al. 1984; Meijlink et al. 1985).

Recent studies have allowed the identification of two functionally independent determinants within the c-fos transcript that specifically target this message for rapid decay (Fort et al. 1987; Rahmsdorf et al. 1987; Kabnick and Housman 1988; Wilson and Treisman 1988; Shyu et al. 1989|. One of the determinants is an AU-rich element (ARE) present in the $3^{\prime}$-untranslated region of $\mathrm{c}$-fos mRNA (Wilson and Treisman 1988; Shyu et al. 1989). The second c-fos instability element, which is located in the protein coding region of the c-fos message (Kabnick and Housman 1988; Shyu et al. 1989), is structurally unrelated to the ARE. Introduction of either of the c-fos instability determinants into the stable $\beta$-globin message markedly reduces its half-life (Shyu et al. 1989). However, deletion of either one of these elements from c-fos mRNA fails to stabilize the c-fos message due to the continued presence of the other instability determinant (Shyu et al. 1989).

Little is yet known about the structural features of the c-fos instability determinants that are important for their function as mRNA destabilizing elements, although the presence of AU-rich sequences in the $3^{\prime}$-untranslated regions of many immediate early gene mRNAs has led to the suggestion that a number of these messages are degraded by a common ARE-dependent 
pathway (Caput et al. 1986; Shaw and Kamen 1986). An intriguing feature of $c-f o s$ mRNA is that shortly after its arrival in the cytoplasm its poly(A) tail undergoes significant shortening (Wilson and Treisman 1988). Deadenylation might therefore be an important event in c-fos mRNA decay.

To investigate the mechanism by which c-fos mRNA is targeted for rapid degradation, we have examined the importance of poly(A) tail removal for c-fos mRNA degradation in growth factor stimulated cells. We have identified two distinct determinants within c-fos mRNA that confer the property of rapid deadenylation on a normally stable message. Remarkably, these two elements correspond directly to the instability determinants present in the coding and $3^{\prime}$-untranslated regions of c-fos mRNA. Quantitative measurements of the rate and extent of deadenylation indicate that the c-fos mRNA instability determinants mediate nearly complete removal of the poly(A) tail before degradation of the transcribed portion of the message begins. Taken together, these results suggest a mechanism for c-fos mRNA degradation whereby functionally independent and structurally distinct instability determinants act in parallel to direct mRNA deadenylation as a critical first step that triggers mRNA decay.

\section{Results}

\section{Deadenylation of c-fos mRNA in NIH-3T3 cells}

To assess the importance of poly(A) tail removal for $\mathrm{c}$-fos mRNA decay, we examined the rate and extent of deadenylation of mouse c-fos mRNA in serum-stimulated NIH-3T3 cells. Transient c-fos transcription was induced by serum stimulation of growth-arrested $3 \mathrm{~T} 3 \mathrm{fi}$ broblasts, and total cytoplasmic RNA was isolated at time intervals. The length of the poly(A) tail present on c-fos mRNA in each sample was determined by Northern blot analysis (Fig. 1). To facilitate this measurement, we subjected the c-fos message to oligonucleotidedirected RNase $\mathrm{H}$ cleavage prior to Northern blotting. For this purpose, we used an oligonucleotide that was complementary to a 20-nucleotide segment of c-fos mRNA located $0.37 \mathrm{~kb}$ upstream of its poly(A) addition site. This procedure resulted in cleavage of $c$-fos mRNA within the segment of its 3 -untranslated region that annealed to the oligonucleotide, thereby releasing a large RNA fragment of $1.7 \mathrm{~kb}$ encompassing the $5^{\prime}$ and central portions of the c-fos message and a small RNA fragment that corresponded to the terminal segment of the c-fos $3^{\prime}$-untranslated region, including the poly(A) tail (Fig. 1). The mobility of a c-fos $3^{\prime}$ fragment that completely lacked a poly $\mid \mathrm{A})$ tail was determined by deadenylating c-fos mRNA in vitro prior to Northern blotting; this was accomplished by annealing oligo(dT) to the poly(A) tail of c-fos mRNA before RNase $\mathrm{H}$ digestion. By comparing the electrophoretic mobility of each $3^{\prime}$ c-fos mRNA fragment with the mobility of the $3^{\prime}$ fragment that had been deadenylated in vitro, it was possible to estimate poly $(\mathrm{A})$ tail lengths (Fig. 2).

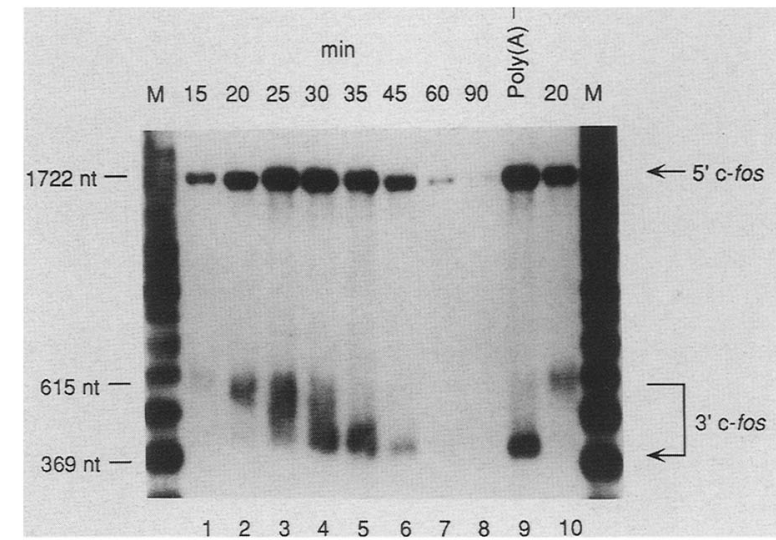

Figure 1. Deadenylation and decay of c-fos mRNA. Total cytoplasmic RNA was isolated at time intervals after serum stimulation of quiescent NIH-3T3 cells, and samples $(8 \mu \mathrm{g})$ were subjected to oligonucleotide-directed RNase $\mathrm{H}$ cleavage and Northern blotting as described in Materials and methods (lanes 1-10). A deadenylated 3' fragment of c-fos mRNA [poly|A |'] was prepared in vitro by annealing the 20-min RNA sample with both the DNA 20-mer and oligo/dT/ $\left.\right|_{12-18}$ prior to RNase $\mathrm{H}$ digestion (lane 9). Lane $M$ contains molecular size markers. Fragments of c-fos mRNA are identified by arrows. Time $(\mathrm{min})$ corresponds to minutes after serum induction.

Using the oligonucleotide-directed RNase $\mathrm{H}$ cleavage procedure, newly synthesized c-fos mRNA was detected in the cytoplasm within $15 \mathrm{~min}$ after serum stimulation. At this time the c-fos $3^{\prime}$ fragment carrying the poly(A) tail was resolved as a sharp band whose mobility indi-

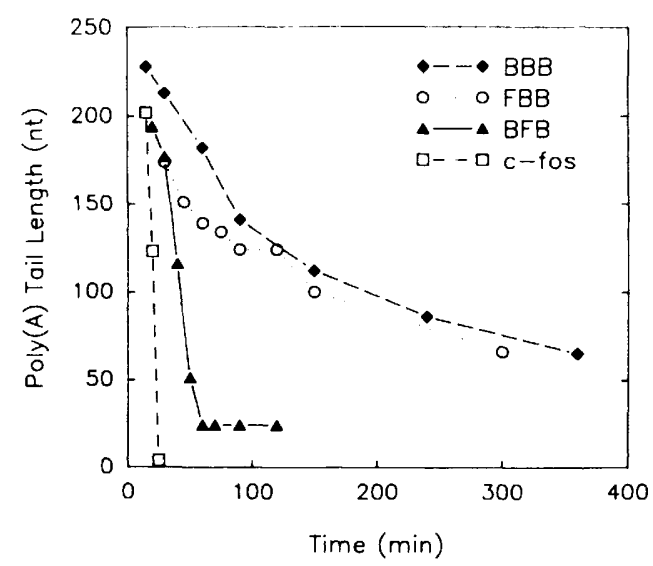

Figure 2. Comparison of the rate and extent of $\operatorname{poly}(A)$ shortening for c-fos, $\beta$-globin, BFB, and FBB mRNA. Poly(A) tail length for c-fos (ㅁ) (Fig. 1), BBB ( ) (Fig. 3), BFB (A) (Fig. 4), and FBB (O) (blot not shown) mRNA is plotted as a function of time after serum stimulation. These tail lengths were calculated from the difference in electrophoretic mobility between each message or fragment thereof and cognate RNA that had been deadenylated in vitro. Mobility differences were measured using the bottom edge of each band as a reference point. The length of poly $(A)$ responsible for the observed differences in mobility was then estimated by comparison with the spacing of bands in a ladder of single-stranded DNA markers that had been electrophoresed in parallel. 
cated that the newly synthesized c-fos mRNA possessed a poly(A) tail with a length of $\sim 200$ nucleotides. By 20 25 min after serum stimulation, the $3^{\prime}$ fragments migrated as a diffuse band. At $25 \mathrm{~min}$, the bottom edge of this band corresponded to the position of poly $(A)^{-}$RNA and the top edge comigrated with RNA having a fulllength poly(A) tail of $\sim 200$ nucleotides. This finding indicates that poly(A) shortening of c-fos mRNA begins within 20 min of serum addition, and that complete removal of the 200-nucleotide poly(A) tail takes as little as $10 \mathrm{~min}$. The diffuse nature of the $3^{\prime}$ fragment in the 25 to 30-min samples most likely reflects the fact that deadenylation of cytoplasmic c-fos mRNA begins before nuclear synthesis of c-fos mRNA stops. Therefore, new c-fos mRNA carrying a full-length poly(A) tail is being delivered to the cytoplasm at the same time that the poly(A) tail is being removed from slightly older c-fos transcripts. This explanation for the diffuse nature of the 3 ' fragment is supported by nuclear run-on transcription assays indicating that $\mathrm{c}$-fos transcription continues until -30 min after serum induction /Greenberg and Ziff 1984; Rivera et al. 1990). Once c-fos transcription ceased, the diffuse $3^{\prime}$ fragment collapsed to a sharper band (3545 min samples) that comigrated with the $3^{\prime}$ fragment of poly $(\mathrm{A})^{-} \mathrm{c}$-fos mRNA. We conclude that within $45 \mathrm{~min}$ of induction, deadenylation of all c-fos mRNA is essentially complete.

During the time that deadenylation took place, the concentration of the $1.7-\mathrm{kb} 5^{\prime}$ segment remained fairly constant. The concentration of this RNA segment then decreased precipitously just after deadenylation reached completion (Fig. 1). Because a similar delay in degradation of the c-fos mRNA body has been observed in cells treated with actinomycin D (Wilson and Treisman 19881, this lag cannot be attributed merely to a brief period of continuing c-fos mRNA synthesis after degradation begins. Instead, this lag in decay suggests that the body of the c-fos message is not degraded until after its poly(A) tail has been removed. We conclude that c-fos mRNA undergoes rapid and complete deadenylation within minutes of its synthesis in serum-stimulated fi- broblasts and that degradation of the c-fos poly(A) tail precedes the decay of the rest of the message.

\section{Deadenylation of $\beta$-globin mRNA in NIH-3T3 cells}

To determine whether rapid and complete removal of the poly $(A)$ tail is a general property of mRNAs synthesized in serum-stimulated cells, we examined the rate and extent of deadenylation of the stable $\beta$-globin message in 3T3 cells. A gene comprising the rabbit $\beta$-globin transcription unit fused to the c-fos promoter (BBB; Shyu et al. 1989/ was transfected into 3T3 cells, and transient synthesis of $\beta$-globin mRNA was induced by treatment of the transfected cells with serum. Because the $0.59-\mathrm{kb}$ length of the $\beta$-globin message upstream of its poly(A) tail is significantly shorter than that of c-fos mRNA (2.1 $\mathrm{kb}$ ), it was possible by Northern blotting to monitor the poly $(A)$ tail length of $\beta$-globin mRNA at various times after induction without the need for prior oligonucleotide-directed RNase $\mathrm{H}$ cleavage of the mRNA.

Synthesis of BBB ( $\beta$-globin) mRNA, like that of $c-f o s$ mRNA, is highly inducible upon serum stimulation; however, in contrast to c-fos mRNA, the $\beta$-globin message is very stable and persists in the cytoplasm for many hours (Shyu et al. 1989). Despite its stability, the $\beta$-globin message underwent slow, highly synchronized poly(A) shortening for at least $6 \mathrm{hr}$ after scrum stimulation (Fig. 3). The rate of poly(A) removal from $\beta$-globin mRNA gradually declined until it ceased $\sim 360$ min after induction (Figs. 2 and 3). When the process of deadenylation stopped, $\beta$-globin mRNA retained a poly $(A)$ tail of $\sim 65$ nucleotides. No change in the length of the $\beta$-globin poly(A) tail could be detected over the next $18 \mathrm{hr}$, during which the $\beta$-globin message remained stable. Thus, while both the labile c-fos message and the stable $\beta$-globin message undergo poly(A) tail removal in serumstimulated fibroblasts, there is a clear difference in the rate and extent of the deadenylation process (Fig. 2). These experiments indicate that sequence determinants within the body of a message control the process of deadenylation and raise the possibility that the rate of mRNA

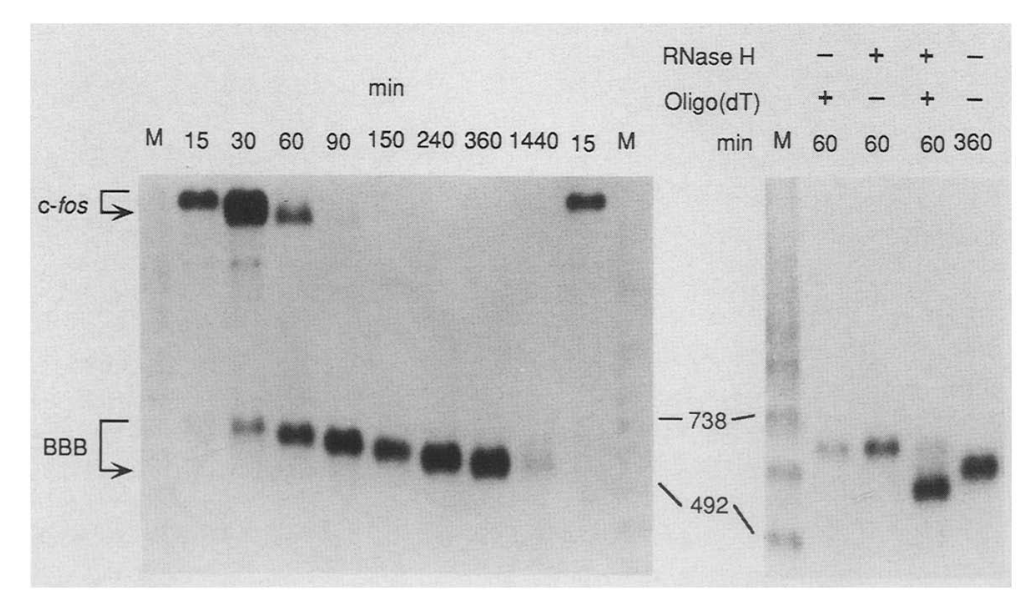

Figure 3. Deadenylation and decay of rabbit $\beta$-globin mRNA. NIH-3T3 cells were transiently transfected with pBBB (Shyu et al. 1989), and total cytoplasmic RNA was isolated at time intervals after serum stimulation. RNA samples $(10 \mu \mathrm{g})$ were analyzed by Northern blotting. (Left) Time course of $\beta$-globin mRNA deadenylation. The RNA blot was hybridized with probes complementary to $\beta$-globin mRNA (BBB), c-fos mRNA, and the molecular size markers (M). Variation in $\beta$-globin mRNA band intensity is due primarily to differences in transfection efficiency. (Right) Comparison with poly $(\mathrm{A})^{-} \beta$-globin mRNA. An RNA sample isolated $60 \mathrm{~min}$ after serum stimulation was treated with oligo(dT $)_{12-18}$ only, RNase $\mathrm{H}$ only, or both and subjected to electrophoresis beside an untreated sample isolated $360 \mathrm{~min}$ after stimulation. The blot was probed with radiolabeled DNA complementary to $\beta$-globin mRNA and the molecular size standards (M). 
decay is determined by both the rate and extent of poly(A) tail removal.

\section{The c-fos-coding region directs fast removal of poly(A)}

To identify these deadenylation determinants, various regions of the c-fos and $\beta$-globin genes were swapped, and the rate and extent of deadenylation of the resulting chimeric transcripts were analyzed. The half-lives of the chimeric messages were also measured to assess whether a strict correlation exists between the lifetime of a message and its rate of deadenylation.

Replacement of the 5 -untranslated region of $\beta$-globin mRNA with that of c-fos (FBB) does not accelerate either degradation (Shyu et al. 1989) or deadenylation of this message (Fig. 2). In contrast, the chimeric BFB message, which contains the 5 -untranslated region of $\beta$-globin, the $\mathrm{c}$-fos coding-region, and the $\beta$-globin 3 '-untranslated region, has a very short half-life $(<20 \mathrm{~min})$ in serumstimulated $3 \mathrm{~T} 3$ cells due to the presence of the strong c-fos coding-region determinant of instability /Shyu et al. 1989). Oligonucleotide-directed RNase H analysis was employed to measure the rate and extent of deadenylation of BFB mRNA transiently synthesized from a c-fos promoter in a stably transfected NIH-3T3 cell line. After RNase $\mathrm{H}$ cleavage and Northern blotting, two BFB mRNA fragments were detected (Fig. 4). The 0.93-kb fragment corresponded to the $5^{\prime}$ segment of BFB mRNA, while the smaller fragment ranging in size from 0.56 to $0.38 \mathrm{~kb}$ represented the $3^{\prime}$ segment, which carries the poly(A) tail. Approximately 20 min after scrum addition, the 3' fragment had a poly (A) tail of $\sim 195$ nucleotides. Thereafter, deadenylation of BFB mRNA proceeded rapidly until $60 \mathrm{~min}$ after serum stimulation. At this point, the BFB 3' fragment possessed a poly(A) tail of $\sim 25 \mathrm{nu}$ cleotides. BFB messages with poly $(\mathrm{A})$ tails shorter than this length were not detected.

Densitometric scanning of the upper band in Figure 4 revealed that the 5 ' segment of BFB mRNA failed to decay significantly until $\sim 30$ min after transcription shutoff (i.e., $60 \mathrm{~min}$ after serum addition). Thereafter, the BFB message decayed rapidly with a half-life of just $14 \mathrm{~min}$. This lag in degradation was not due to ongoing synthesis of BFB mRNA or to continued export of this mRNA from the nucleus, as new BFB messages with full-length poly(A) tails were no longer detectable as early as $40 \mathrm{~min}$ after serum stimulation. The rapidity with which BFB transcription ceases is consistent with previous reports that transcription from the c-fos promoter is completely shut off within $30 \mathrm{~min}$ of senum stimulation of $3 \mathrm{~T} 3$ cells (Greenberg and Ziff 1984; Rivera et al. 1990). Comparison of the lag in degradation of the $5^{\prime}$ segment of BFB mRNA with the time course of poly(A) loss from the $3^{\prime}$ end of this message indicates that decay of the body of the BFB message does not begin until poly(A) removal is essentially complete (Fig. 4). We conclude from this experiment that introduction of the c-fos coding region into $\beta$-globin mRNA confers both a short half-life and the property of rapid and nearly complete deadenylation on the normally highly stable $\beta$-globin message. Further-
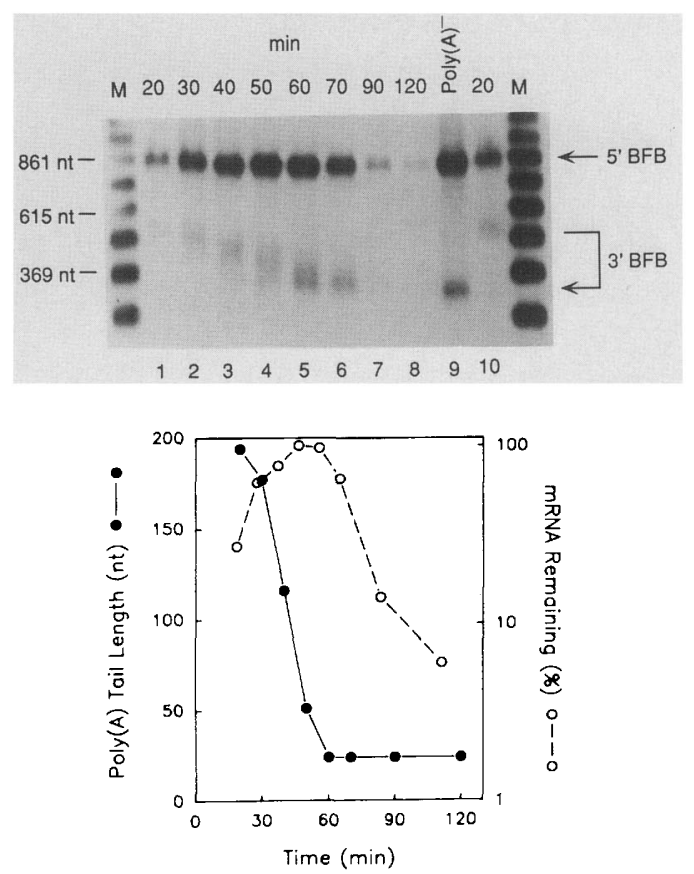

Figure 4. Deadenylation and decay of BFB mRNA. An NIH3T3 cell line stably transfected with pBFB was established, and total cytoplasmic RNA was isolated at time intervals after serum stimulation. RNA samples $(7 \mu \mathrm{g})$ were subjected to oligonucleotide-directed RNase $\mathrm{H}$ cleavage and Northern blotting (lanes 1-10). (Top) RNA blot. The $5^{\prime}$ and $3^{\prime}$ fragments of BFB mRNA are indicated. A deadenylated 3 ' fragment of BFB mRNA [poly(A) | was prepared in vitro by annealing the 20-min RNA sample with both the DNA 20 -mer and oligo $(\mathrm{dT})_{12-18}$ prior to RNase $\mathrm{H}$ digestion (lane 9). (Bottom) Composite plot of the time course of the deadenylation and decay of BFB mRNA. Poly $(A)$ tail length $(O)$ was determined as in Fig. 2. BFB mRNA remaining $(O)$ was quantitated by densitometric analysis of the intensity of each band representing the $5^{\prime}$ segment of BFB mRNA.

more, poly $(\mathrm{A})$ tail removal appears to be the first step in the BFB mRNA decay process triggered by the c-fos coding region.

\section{The deadenylation and instability determinants within the c-fos coding region coincide}

To establish whether the determinant within the c-fos coding-region that directs deadenylation corresponds to the previously identified instability determinant in this segment of c-fos mRNA, smaller portions of the c-fos coding-region were tested for their ability to direct both rapid deadenylation and degradation of $\beta$-globin mRNA. We have recently localized a major determinant of mRNA instability to a $0.32-\mathrm{kb}$ sequence within the $\mathrm{c}$-fos coding-region (A.-B. Shyu et al., in prep.). This $0.32-\mathrm{kb}$ RNA segment, when introduced in-frame into the $\beta$-globin (BBB) coding region, reduced the half-life of $\beta$-globin mRNA from $>24 \mathrm{hr}$ to $\sim 40 \mathrm{~min}$ (Fig. 5 ). In contrast, no such destabilization of $\beta$-globin mRNA is observed upon insertion of a $0.31-\mathrm{kb}$ coding-region seg- 

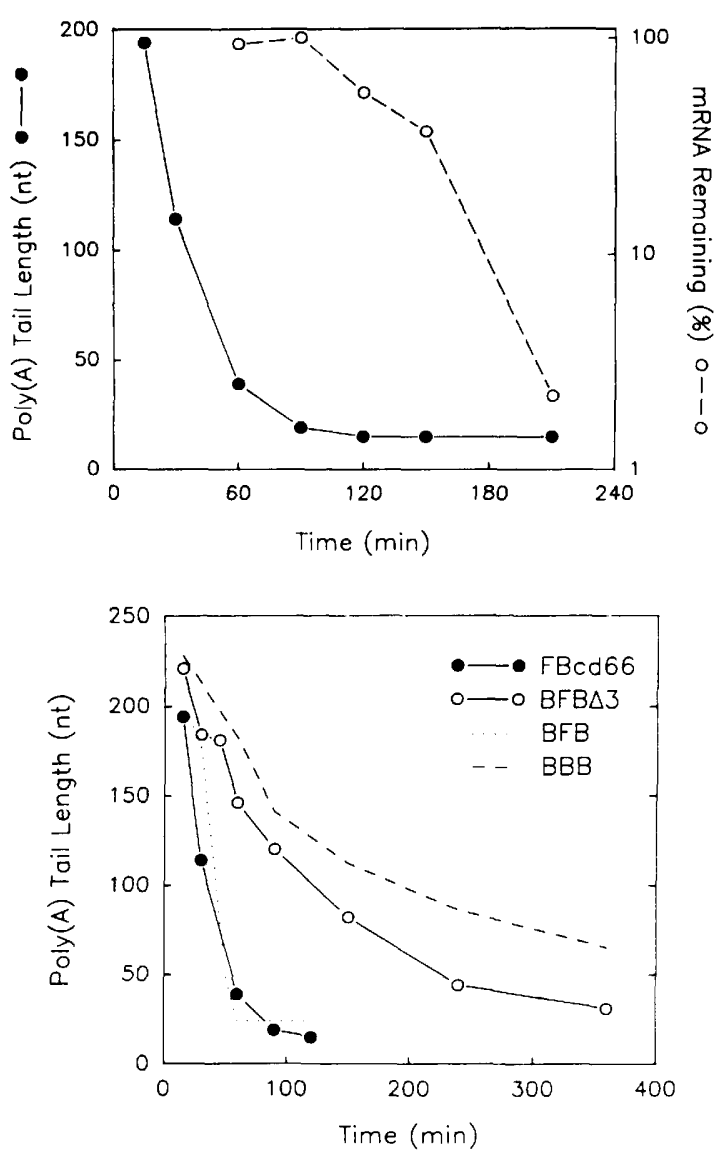

FBcd66

BFB.13

Figure 5. Deadenylation and decay of FBcd 66 and $B F B \Delta 3$ mRNA. (Top) A composite plot of the time course of the deadenylation and decay of FBcd66 mRNA is shown. NIH-3T3 cells were transiently co-transfected with pFBcd66 and $\mathrm{pSV} \alpha$ l (Shyu et al. 1989), and total cytoplasmic RNA was isolated at time intervals after serum stimulation. RNA samples $(10 \mu \mathrm{g})$ were analyzed by Northern blotting as in Fig. 3 and by RNase protection (see Materials and methods). Poly(A) tail lengths (O) were determined as in Fig. 2. FBcd66 mRNA remaining $(O)$ was determined by densitometric analysis of the autoradiogram obtained from the RNase protection experiment (not shown), using $\alpha$-globin mRNA as an internal standard to allow correction for variations in transfection efficiency (Shyu et al. 1989). (Middle) Poly (A) tail length for BFB $\Delta 3$ (O) (blot not shown) and FBcd66 $(-)$ mRNA is plotted as a function of time after serum stimulation. Also plotted is the time course of deadenylation of BFB $(\ldots)$ and BBB ( $\beta$-globin) (- . - ) mRNA (see Fig. 2). Poly(A) tail lengths were calculated as in Fig. 2. (Bottom) Diagrams of FBcd 66 and BFB $\Delta 3$ mRNA. (Lines) $\beta$-globin UTRs; (open rectangles) $c$-fos coding-region segments; (solid rectangles) $\beta$-globin coding-region segments; $\left(A_{n}\right)$ poly $(A)$.

ment from the stable glyceraldehyde-3-phosphate dehydrogenase message (data not shown). The rate and extent of deadenylation of the hybrid message (FBcd66) containing the $0.32-\mathrm{kb}$ c-fos coding-region segment were nearly the same as those for BFB mRNA, and significant decay of the body of this message was observed only after the
poly(A) tail had been almost completely removed (Fig. 5). These results indicate that the c-fos deadenylation and instability determinants are present within the same $0.32-\mathrm{kb}$ region of the $\mathrm{c}-$ fos coding-region. The colocalization of these two determinants supports the idea that the coding-region determinant initiates message degradation by promoting the rapid removal of the poly(A) tail.

To confirm that the rapid and complete deadenylation of BFB mRNA is correlated with its rapid degradation, we then asked whether a mutation in BFB mRNA that reduces its rate of degradation also impedes the deadenylation process. BFB $\Delta 3$ mRNA contains a $0.42-\mathrm{kb}$ coding-region deletion that retards degradation, resulting in a half-life $(200 \mathrm{~min})>10$ times longer than that of intact BFB mRNA (Shyu et al., in prep.). Analysis of the BFB $\triangle 3$ mRNA deadenylation process revealed that the slow degradation rate caused by this deletion is accompanied by a substantial decrease in the rate and extent of deadenylation (Fig. 5).

To extend the correlation between deadenylation and decay of BFB mRNA, we asked whether changes in gene expression and cell growth state that alter the efficacy of the coding-region determinant as a destabilizing element might also affect the deadenylation process. Previous studies have established that mutant c-fos mRNAs bearing a deletion of just the $3^{\prime}$ AU-rich instability determinant are fairly stable when constitutively synthesized in actively growing fibroblasts (Fort et al. 1987; Rahmsdorf et al. 1987). These results are quite different from those we obtained with growth factor-inducible messages in serum-stimulated fibroblasts, where we found that deletion of the ARE has little effect on the half-life of c-fos mRNA due to the continued presence of the coding-region instability determinant. A possible explanation for this discrepancy is that the coding-region determinant might not function effectively when c-fos mRNA is continuously synthesized in growing cells.

In support of this idea, we find that while BFB mRNA decays with a short half-life of $\sim 20 \mathrm{~min}$ in serum-stimulated NIH-3T3 cells, it is much more stable when constitutively synthesized in actively growing NIH-3T3 cells, where it decays with a long half-life of $300 \pm 80$ min. In these experiments, the decay and deadenylation of BFB mRNA in actively growing fibroblasts were examined by fusing the BFB gene to an SV40 enhancer to allow synthesis of BFB mRNA in the absence of serum stimulation. The half-life and deadenylation of the constitutively synthesized BFB (SV-BFB) message were monitored at time intervals after transcription was inhibited with 5,6-dichloro-1- $\beta$-D-ribofuranosylbenzimidazole (DRB). Concomitant with the reduced degradation rate of SV-BFB mRNA in these cells, the rate and extent of deadenylation of this mRNA were also found to decrease (Fig. 6). The slow rate of SV-BFB mRNA degradation observed in this experiment was not due to the use of the inhibitor DRB, as the addition of DRB to serum-stimulated fibroblasts at the peak of BFB mRNA synthesis has little effect on the half-life of the BFB message (Shyu et al. 1989). Taken together, these results support the proposals that the instability determinant in the c-fos cod- 

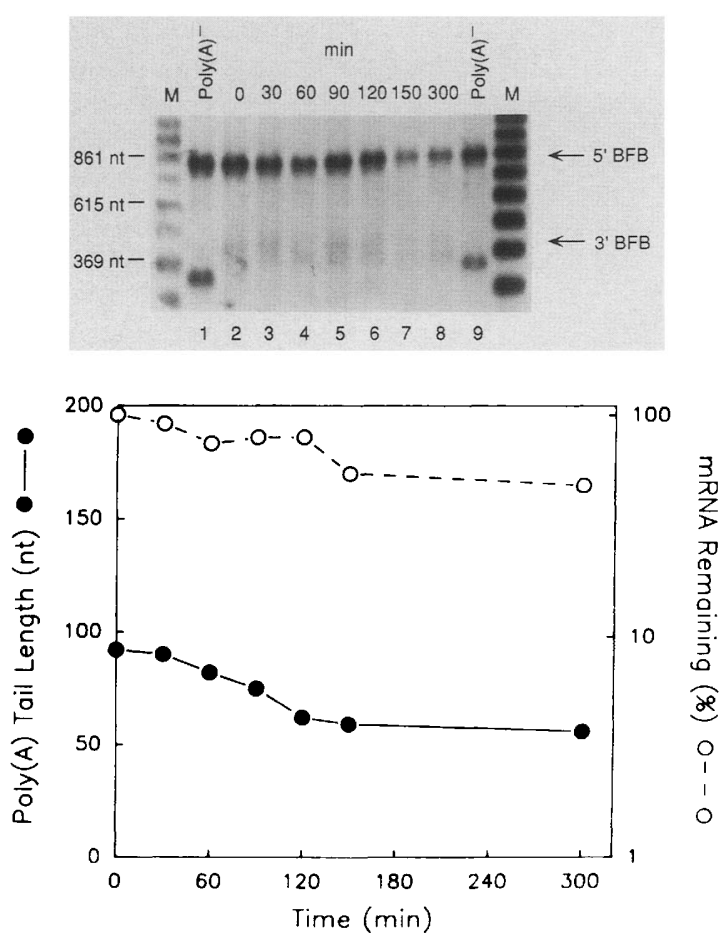

Figure 6. Deadenylation and decay of SV-BFB mRNA in actively growing fibroblasts. NIH-3T3 cells were transiently cotransfected with pSV-BFB and pSV $\alpha 1$ (Shyu et al. 1989), and total cytoplasmic RNA was isolated from actively growing cells at time intervals after transcription inhibition with DRB (30 $\mu \mathrm{g} / \mathrm{ml})$. RNA samples were analyzed by oligonucleotidedirected RNase $\mathrm{H}$ cleavage and Northern blotting as in Fig. 4 and by RNase protection as in Fig 5. (Top) RNA blot. The $5^{\prime}$ and 3 ' fragments of SV-BFB mRNA are indicated. Deadenylated SVBFB mRNA (lanes 1 and 9) was prepared as in Fig. 4. (Bottom) A composite plot of the time course of decay and deadenylation of SV-BFB mRNA is shown. Poly(A) tail lengths $(0)$ were determined as in Fig. 2, except that the middle of each band was used as a reference point for estimating the average tail length of these messages, which are highly heterogeneous in age. SV-BFB mRNA remaining $(O)$ was determined by RNase protection (autoradiogram not shown).

ing-region coincides with the coding-region deadenylation determinant and that this element mediates mRNA decay by first promoting rapid removal of the poly $(A)$ tail. The failure of the coding-region instability determinant to function when the BFB message is constitutively expressed in growing cells may explain why simple deletion of the c-fos ARE is sufficient both to stabilize c-fos mRNA continuously synthesized in growing cells and to enhance the transforming potential of a c-fos proto-oncogene fused to a constitutive promoter (Meijlink et al. 1985 |.

Insertion of the $c$-fos $A U$-rich element into $\beta$-globin mRNA destabilizes the $\beta$-globin message and increases its rate and extent of deadenylation

To determine whether the ability of the c-fos codingregion determinant to confer rapid deadenylation on $\beta$-globin mRNA represents a general mechanism by which instability elements function, we asked whether insertion of the c-fos AU-rich element into the $3^{\prime}$ untranslated region of $\beta$-globin mRNA (to generate $\mathrm{BBB}+\mathrm{ARE}$ mRNA) affects its rate and extent of deadenylation. BBB + ARE mRNA decays much more quickly than the wild-type $\beta$-globin message (Fig. 7; Shyu et al. 1989), and this chimeric message also displays an enhanced rate of deadenylation (Fig. 8). In contrast to the $\beta$-globin message, which still retains $>60$ nucleotides of its poly(A) tail $24 \mathrm{hr}$ after synthesis (Fig. 2), deadenylation of the BBB + ARE message was virtually complete within $150 \mathrm{~min}$, with $<25$ nucleotides of its poly $(\mathrm{A})$ tail
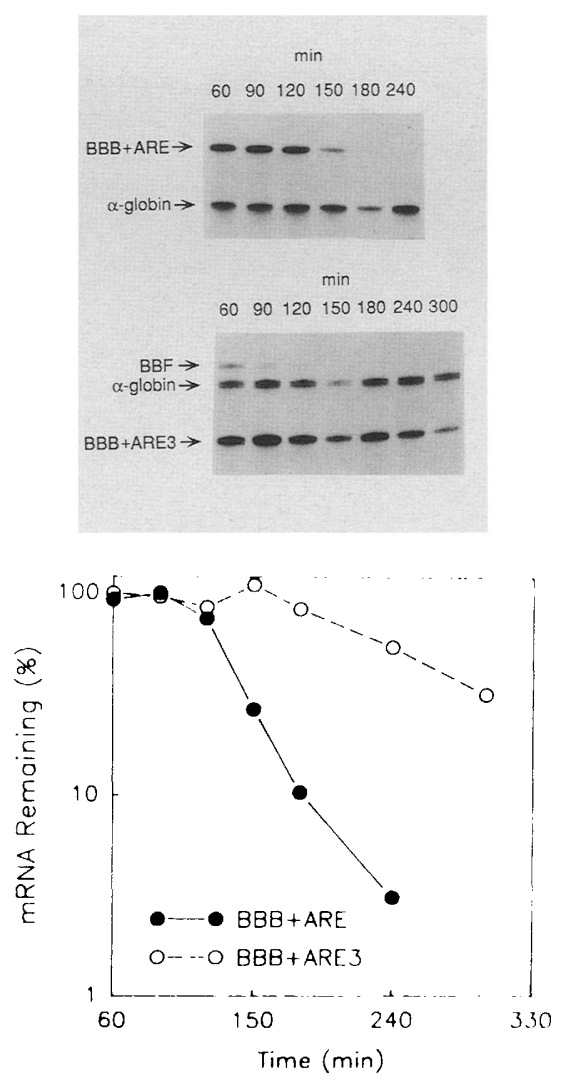

Figure 7. Decay of $B B B+A R E$ and $B B B+A R E 3$ mRNA. NIH-3T3 cells were transiently transfected with pSV $\alpha 1$ and either $\mathrm{pBBB}+\mathrm{ARE}($ top $)$ or $\mathrm{pBBB}+\mathrm{ARE} 3$ and $\mathrm{pBBF}$ (middle), and total cytoplasmic RNA was isolated at time intervals after serum stimulation. (Top) RNase protection analysis of $\mathrm{BBB}+\mathrm{ARE}$ mRNA decay. RNA probe fragments protected by $\mathrm{BBB}+\mathrm{ARE}$ mRNA and $\alpha$-globin mRNA (an internal standard; Shyu et al. 1989) are indicated. (Middle) RNase protection analysis of $B B B+A R E 3$ mRNA decay. RNA probe fragments protected by BBB + ARE3, BBF, and $\alpha$-globin mRNA are indicated. The rapid decay of BBF mRNA, which consists of the 5 '-untranslated region and coding region of $\beta$-globin mRNA fused to the c-fos 3 '-untranslated region (Shyu et al. 1989), shows that mRNA containing an intact c-fos ARE can be degraded quickly in the same cells in which BBB + ARE3 mRNA decays slowly. (Bottom) Semilogarithmic plot of the decay of $\mathrm{BBB}+\mathrm{ARE}(O)$ and $\mathrm{BBB}+\mathrm{ARE} 3$ (O) mRNA. The measured half-life of $\mathrm{BBB}+\mathrm{ARE} 3 \mathrm{mRNA}$ was $149 \pm 34 \mathrm{~min}$. 
remaining at that time (Fig. 8). After transcription ceased at $\sim 30 \mathrm{~min}$ poststimulation, there was a $90-\mathrm{min}$ time lag before the BBB + ARE message decayed. During this period, deadenylation of $\mathrm{BBB}+\mathrm{ARE}$ mRNA proceeded, but the concentration of this mRNA remained fairly constant (Fig. 7). As observed for the BFB and wild-type c-fos messages, decay of BBB + ARE mRNA was not evident until its poly(A) tail had been shortened to $<30$ nucleotides. This experiment demonstrates that inser-
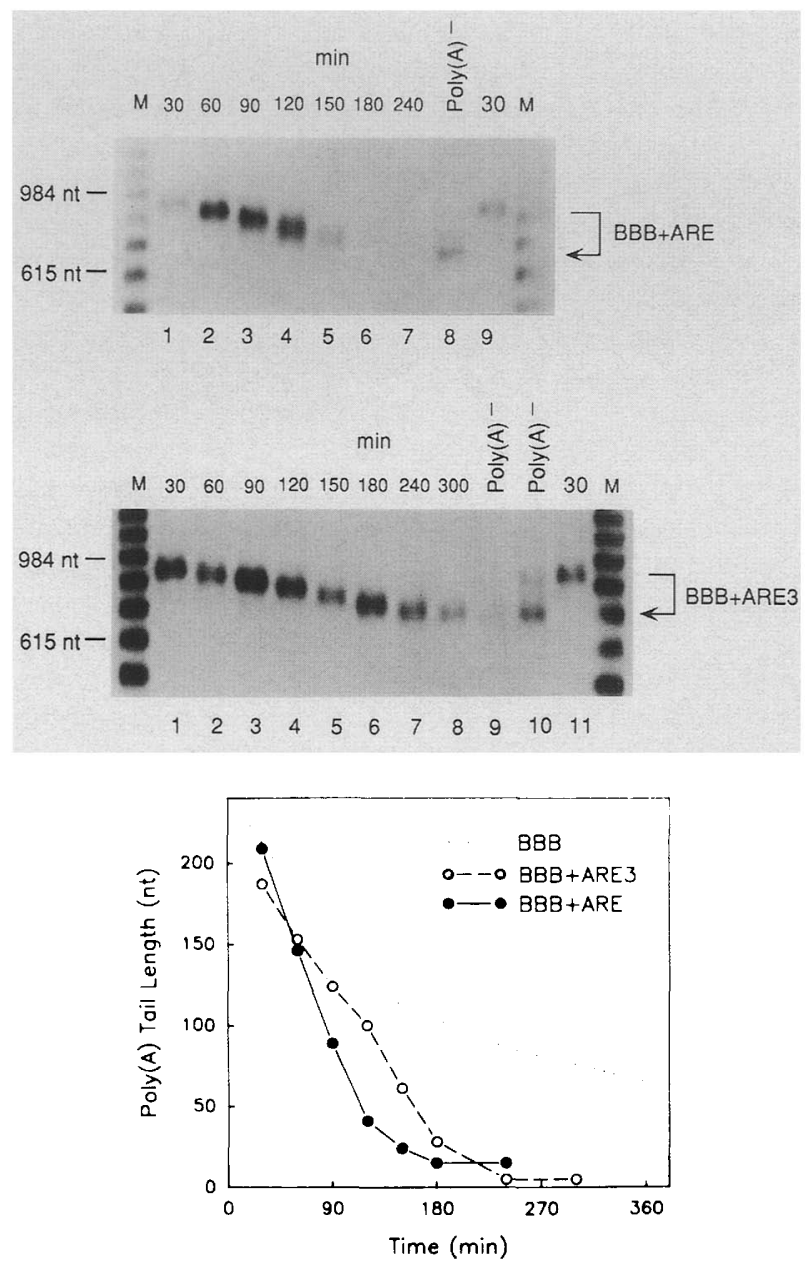

Figure 8. Deadenylation of $B B B+A R E$ and $B B B+A R E 3$ mRNA. Northern blot analysis of the deadenylation of $\mathrm{BBB}+\mathrm{ARE}$ mRNA (top) and BBB + ARE3 mRNA (middle). RNA samples isolated at time intervals after serum induction were the same as those used for the experiments in Fig. 7. The blots were probed with radiolabeled DNA complementary to $\beta$-globin mRNA. Bands corresponding to BBB + ARE (top) and $\mathrm{BBB}+\mathrm{ARE} 3$ (middle) $\mathrm{mRNA}$ are indicated. Variation in band intensity is due in part to differences in transfection efficiency. Deadenylated mRNA [poly|A) ] was prepared in vitro by treating RNA samples isolated 30 min (lane 8, top; lane 10, middle) or $300 \mathrm{~min}$ (lane 9, middle) after serum stimulation with oligo(dT) $)_{12-18}$ and RNase $\mathrm{H}$. (Bottom) Poly(A) tail length for $\mathrm{BBB}+\mathrm{ARE}(O)$ and BBB + ARE3 (O) mRNA is plotted as a function of time after serum stimulation. Also plotted is the time course of deadenylation of BBB ( $\beta$-globin) mRNA $\mid \ldots$ ) (see Fig. 2). Poly $(\mathrm{A})$ tail lengths were calculated as in Fig. 2. tion of the c-fos ARE into $\beta$-globin mRNA accelerates deadenylation and subsequent decay of the message. We conclude that introduction of either of the two c-fos instability determinants can independently trigger degradation of heterologous mRNA by first promoting removal of the poly(A) tail. The discovery of multiple independent elements in c-fos mRNA that direct rapid poly(A) tail removal explains the observation of Wilson and Treisman (1988) that deletion of the ARE from c-fos mRNA retards its deadenylation only slightly.

The AUUUA motif within the ARE is not essential for rapid deadenylation

We then asked whether it is the general AU richness or a particular sequence feature of the ARE that is crucial for its destabilizing activity. Like other destabilizing AREs known to function as mRNA destabilizers (Shaw and Kamen 1986), the c-fos ARE contains multiple copies of the pentanucleotide sequence motif AUUUA 15'-UUUUAUUGUGUUUUUAAUUUAUUUAUUAAG AUGGAUUCUCAGAUAUUUAUAUUUUUAUUUUA UUUUUUU-3'). All three AUUUA pentanucleotides in the c-fos ARE were disrupted by single U-to-A point mutations to preserve the AU richness of the element while altering its sequence. The resulting mutant ARE (ARE3: 5'-UUUUAUUGUGUUUUUAAUAUAUUAAUUAAG AUGGAUUCUCAGAUAUUAAUAUUUUUAUUUUA UUUUUUU-3') was tested for its ability to facilitate the rapid deadenylation and degradation of $\beta$-globin mRNA. Remarkably, this subtle change in the sequence of the ARE dramatically inhibited its ability to destabilize the $\beta$-globin message (Fig. 7). Insertion of ARE3 instead of the wild-type ARE into the $3^{\prime}$-untranslated region of $\beta$-globin mRNA increased the half-life of $\mathrm{BBB}+\mathrm{ARE}$ mRNA approximately fivefold.

In contrast to its effect on the degradation rate of BBB + ARE mRNA, replacement of the ARE with ARE3 had only a small effect on the rate and extent of deadenylation of BBB + ARE mRNA (Fig. 8). The ability of a subtle mutation to impede mRNA degradation without substantially affecting rapid deadenylation suggests that while removal of the poly(A) tail may be an important first step in fast mRNA decay, the role of the AUUUA pentanucleotides within the ARE may be to facilitate subsequent degradation of the body of the BBB + ARE message.

\section{Destabilization of $\beta$-globin mRNA by insertion of $a$ nonsense codon does not involve accelerated deadenylation}

While both the c-fos ARE and the instability determinant in the c-fos coding-region mediate rapid mRNA decay by first facilitating removal of the poly(A) tail, the process of deadenylation is not always a prerequisite for the degradation of labile transcripts. In addition to destabilization by insertion of instability determinants from labile messages, $\beta$-globin mRNA can also be destabilized by mutations in its coding sequence that result in 
premature termination of translation (Kinniburgh et al. 1982). When a nonsense codon was introduced into $\beta$-globin mRNA expressed from a c-fos promoter, the half-life of the resulting BBB36 message in serum-stimulated fibroblasts fell from $>24 \mathrm{hr}$ to only $72 \mathrm{~min}$. While the introduction of this translation termination signal at codon 36 was highly destabilizing, it had little effect on the rate or extent of deadenylation of $\beta$-globin mRNA. BBB36 mRNA lost its poly(A) tail very slowly (Fig. 9) at a rate nearly identical to that seen for wild-type $\beta$-globin mRNA (Figs. 3 and 9). At 300 min after serum stimulation, when $\mathrm{BBB} 36 \mathrm{mRNA}$ still retained $>50$ nucleotides of poly(A), $90 \%$ of the BBB36 mRNA had already been degraded. Taken together, these studies indicate that at least two distinct mechanisms are utilized in growth factor-stimulated cells to degrade labile messages.

\section{Discussion}

Although 20 years have passed since the discovery that nearly all eukaryotic messages carry a $3^{\prime}$ poly(A) tail (Kates 1970; Lim and Canellakis 1970), the primary biological function of poly(A) has remained elusive. It has been suggested that the poly(A) tail might control mRNA processing, regulate mRNA transport from the nucleus to the cytoplasm, affect the efficiency of mRNA translation, or protect mRNA from degradation (for reviews, see Brawerman 1981; Bernstein and Ross 1989; Jackson and Standart 1990).

Several recent studies lend increased support to the proposal that one crucial function of the poly(A) tail is in the regulation of mRNA decay. For example, in some cases the presence of a poly $(A)$ tail at the $3^{\prime}$ end of a transcript has been found to stabilize the message (Mar-

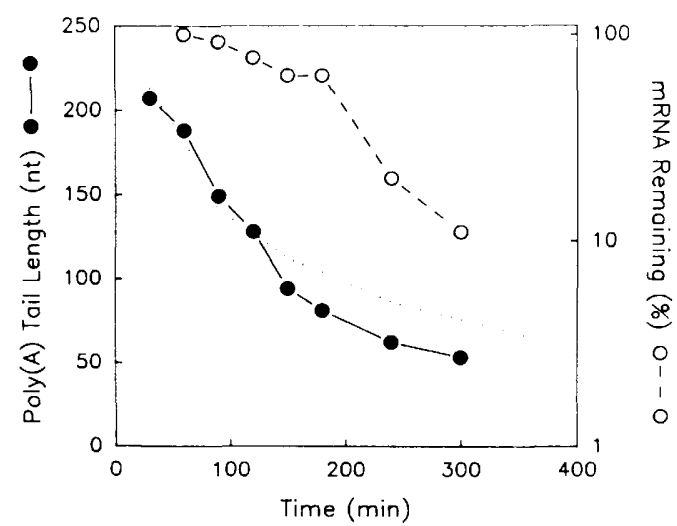

Figure 9. Deadenylation and decay of BBB36 mRNA. NIH-3T3 cells were transiently transfected with plasmids pBBB36 and $\mathrm{pSV} \alpha 1$, and total cytoplasmic RNA was isolated at time intervals after serum stimulation. RNA samples were analyzed by RNase protection and Northern blotting (autoradiograms not shown/ as in Figs. 7 and 8 . A composite plot of the time course of the decay $(O)$ and deadenylation (O) of BBB36 mRNA is shown. Poly(A) tail lengths (O) were calculated as in Fig. 2. For comparison, the time course of deadenylation of BBB mRNA is also plotted $(. .$.$) .$ baix et al. 1975; Drummond et al. 1985; Levine et al. 1987). In addition, the short-lived transcripts of two proto-oncogenes, c-fos and c-myc, are rapidly deadenylated prior to being degraded (Fig. 1; Brewer and Ross 1988; Wilson and Treisman 1988; Swartout and Kinniburgh 1989|. These findings have led these investigators to suggest that poly $(A)$ can protect mRNA from degradation.

In this report we have examined the rate and extent of deadenylation of the labile c-fos and stable $\beta$-globin messages in growth factor-stimulated fibroblasts. Our experiments demonstrate that both of these mRNAs undergo poly(A) shortening immediately after their arrival in the cytoplasm, but with strikingly different kinetics. The marked disparity in the lifetimes of these two transcripts correlates with the considerable difference in the stability of their poly(A) tails, a finding that suggests a causal relationship for c-fos mRNA between deadenylation and rapid decay.

These observations imply a dynamic interaction between the transcribed portion of a message and its poly(A) tail and suggest that structural features of mRNA can function to stimulate or retard deadenylation. By mapping the determinants within the c-fos message that mediate rapid deadenylation, we have demonstrated that these elements coincide with previously characterized determinants in the protein coding region and 3 -untranslated region that are responsible for the instability of c-fos mRNA. For labile messages containing either a functional $c$-fos coding-region element or the c-fos ARE, degradation of the transcribed portion of the message does not begin until after deadenylation is virtually complete. Taken together, these findings demonstrate that two structurally distinct destabilizing elements interact independently with the $\operatorname{poly}(\mathrm{A})$ tail to facilitate its removal as a crucial first step controlling c-fos mRNA decay.

The finding that the c-fos ARE and coding-region determinant, which are structurally unrelated, both initiate the process of mRNA decay by directing removal of the poly(A) tail suggests that deadenylation may be a general mechanism by which a variety of mRNA instability elements function. How these destabilizing elements facilitate deadenylation of mRNA remains to be established. The coding-region instability element and the ARE may bind to cytoplasmic factors that catalyze poly $(A)$ digestion. Such a deadenylation factor could be a poly $(A)$-specific ribonuclease or a protein that exposes the $\operatorname{poly}(A)$ tail to ribonuclease digestion. Although deadenylation appears to be a key step in mRNA degradation mediated by the c-fos ARE and coding-region element, the mechanisms by which these destabilizing elements function are likely to be somewhat different in detail, given the lack of a substantial AU-rich segment in the c-fos coding region and the differential effect of transcription inhibitors on decay mediated by these two elements (Shyu et al. 1989).

While it is likely that deadenylation is an important step in the degradation of a variety of labile mammalian messages, poly(A) tail removal is clearly not the only 
cellular mechanism for initiating rapid mRNA degradation. Destabilization of $\beta$-globin mRNA by a mutation that causes premature translation termination (BBB36) does not accelerate the slow deadenylation rate of this message, whose transcribed portion is rapidly degraded despite a poly(A) tail length $>50$ nucleotides. Apparently, premature termination of translation can target mRNA for degradation by an alternative pathway that does not require complete deadenylation.

Multiple copies of the pentanucleotide AUUUA are present in several natural AU-rich elements that have been shown to function as mRNA destabilizers (Shaw and Kamen 1986; Fort et al. 1987; Jones and Cole 1987; Rahmsdorf et al. 1987; Wilson and Treisman 1988; Shyu et al. 1989; Whittemore and Maniatis 1990). Although intact AUUUA pentanucleotides are not essential for deadenylation mediated by the c-fos ARE, disruption of these sequence motifs by point mutagenesis impairs the ability of the c-fos ARE to effect rapid mRNA degradation. These findings suggest that the ARE is involved in two distinct steps in the mRNA decay process. During the first step, which is AUUUA-independent, the ARE interacts with the poly $(\mathrm{A})$ tail and causes its rapid and nearly complete removal. The second critical step in ARE-mediated decay is the subsequent degradation of the transcribed portion of the message, which appears to be AUUUA dependent.

A number of mechanisms can be envisioned to explain how deadenylation triggers rapid c-fos mRNA decay. One possibility is that poly $(\mathrm{A})$ tail loss may expose the $3^{\prime}$ end of the message to $3^{\prime}$-exonuclease attack. This interpretation is consistent with the discovery in mammalian cells of a cytoplasmic $3^{\prime}$ exoribonuclease that in vitro readily degrades mRNA not protected at its $3^{\prime}$ end by a complex of poly(A) and poly(A)-binding protein (PABP) (Bernstein et al. 1989). In eukaryotic cells, PABP is believed to coat all mRNA poly(A) tails that are at least 12 nucleotides long, at a packing density of one PABP molecule per 27 adenylate residues (Baer and Kornberg 1980; Sachs et al. 1987). A possible role for this protein in impeding degradation of c-fos mRNA is suggested by our finding that poly(A) tails shorter than 15 nucleotides are not observed during degradation of BFB, FBcd66, and $\mathrm{BBB}+\mathrm{ARE}$ mRNA. This finding could be explained if BFB, FBcd66, and BBB + ARE messages with poly(A) tails too short to bind PABP are such extremely labile degradation intermediates that they fail to accumulate to a detectable concentration. In contrast, the longevity of $\beta$-globin mRNA may result in part from the fact that for at least $24 \mathrm{hr}$ after its synthesis this message retains a poly(A) tail long enough to bind two molecules of PABP.

Nevertheless, the degradation mechanism of deadenylated c-fos mRNA may be more complex than implied by a model that simply invokes 3 '-exonuclease digestion, as the absence of a poly(A) tail or of bound PABP does not necessarily target a message for rapid degradation (Sehgal et al. 1978; Krowczynska et al. 1985; Galili et al. 1988; Shapiro et al. 1988; Sachs and Davis 1989|. For example, $\mathrm{BBB}+\mathrm{ARE} 3$ mRNA is degraded rather slowly even after its poly(A) tail has been completely removed. If a $3^{\prime}$ exonuclease does play a general role in the degradation of deadenylated transcripts, then there must be RNA structures or RNA-binding proteins that control the susceptibility of deadenylated messages to 3 '-exonuclease attack. This prediction is supported by our finding that the AUUUA sequence elements in the c-fos ARE appear to facilitate further digestion of deadenylated $\mathrm{BBB}+\mathrm{ARE}$ mRNA.

In summary, our data indicate that two structurally distinct determinants of mRNA instability mediate rapid deadenylation as the first step in c-fos mRNA decay. As such, deadenylation appears to be a critical regulatory event that controls c-fos gene expression by triggering the degradation of its RNA transcript. These findings provide important insights into the overall mechanism of c-fos mRNA degradation and suggest an experimental basis for identifying cellular factors that participate in this process.

\section{Materials and methods \\ Cell culture and DNA transfection}

Culturing, serum stimulation, and transient transfection of mouse NIH-3T3 cells were as described previously (Shyu et al. 1989). A stably transfected 3T3 cell line containing the BFB gene was prepared as described (Rivera et al. 1990).

\section{Analysis of mRNA decay and deadenylation}

Extraction of total cytoplasmic RNA from transfected cells and measurement of mRNA decay rates by RNase protection analysis have been described previously (Shyu et al. 1989).

For RNase protection analysis, a number of different probes were used. A 272-nucleotide RNA probe derived from EcoRIlinearized plasmid pT7 $\alpha 181$ was employed to detect human $\alpha$-globin mRNA in cytoplasmic RNA samples from cells cotransfected with $\mathrm{pBBF}, \mathrm{pBBB}+\mathrm{ARE} 3$, and $\mathrm{pSV} \alpha 1$. This probe is complementary to a 181 -nucleotide segment within the second exon of $\alpha 1$-globin mRNA. In all other RNase protection experiments, $\alpha$-globin mRNA was detected using a probe derived from plasmid pSP6 $\alpha 133$ (Treisman 1985; Shyu et al. 1989). To detect BBF and BBB + ARE3 mRNA, a 238-nucleotide RNA probe was used. This probe, which was transcribed from BamHI-linearized plasmid pT7BBF by T7 RNA polymerase, was complementary to a 208-nucleotide segment within the last exon of BBF mRNA and to a 138-nucleotide mRNA segment within the last exon of BBB + ARE3 mRNA. To detect SV-BFB and $B F B \triangle 3$ mRNA, an RNA probe derived from plasmid pSP6BFB was used (Shyu et al. 1989). FBcd66, BBB + ARE, and $\mathrm{BBB} 36$ mRNA were detected with an RNA probe derived from plasmid pT7BG (Shyu et al. 1989).

RNase $\mathrm{H}$ treatment of cytoplasmic mRNA was performed according to the methods of Kleene et al. (1984) and Brewer and Ross (1988). Samples of total cytoplasmic RNA (7-10 $\mu \mathrm{g}$ ) were annealed with either of two synthetic oligodeoxynucleotides $(0.3 \mu \mathrm{g})$. One oligonucleotide $\left(5^{\prime}\right.$-CGCTATTGCCAGGAACA CAG-3') was complementary to a sequence 368 nucleotides upstream of the poly(A) addition site of mouse c-fos mRNA. The other (5'-TGTCTGGCACGGAGCGGGCT-3') was complementary to a sequence 360 nucleotides upstream of the poly(A) addition site of BFB mRNA. For in vitro deadenylation of mRNA, oligo(dT $)_{12-18}(0.3 \mu g$; Pharmacia) was used. 
Northern blot analysis of cytoplasmic mRNA was performed as described previously (Bartel et al. 1989). A 123-nucleotide single-stranded DNA ladder (BRL) was included to provide a set of molecular size markers. Electrophoresis was on $1.2-1.4 \%$ agarose/formaldehyde gels. Gene-specific DNA probes were prepared by the method of random oligonucleotide priming (Feinberg and Vogelstein 1983).

\section{Plasmid constructions}

When necessary, DNA with $5^{\prime}$ - or $3^{\prime}$-protruding ends was treated with T4 DNA polymerase to make the ends blunt.

Plasmid pFBcd66 encodes a chimeric message consisting of a hybrid $\beta$-globin/c-fos/ $\beta$-globin-coding region flanked by the $\beta$-globin $5^{\prime}$ - and $3^{\prime}$-untranslated regions. A 0.49 -kb StuI-BglII fragment was excised from plasmid pBFB (Shyu et al. 1989) and replaced with a $0.91-\mathrm{kb} N c o$ I (filled-in)-BglII fragment of pSVB10 (Shyu et al. 1989) that spanned part of the second exon, all of the second intron, and part of the third exon of the rabbit $\beta$-globin gene. A 1.36-kb MluI-Sphl (blunt) fragment of the resulting plasmid was then replaced with a $0.59-\mathrm{kb}$ MluI-HincII fragment of pBBB4 (Shyu et al. 1989) to generate pFBcd66.

To generate plasmid pBFB $\Delta 3$, a $0.49-\mathrm{kb}$ StuI-BgIII (filled-in) fragment encoding the Fos carboxyl terminus was excised from plasmid pBFB (Shyu et al. 1989) and replaced by linker-tailing (Lathe et al. 1984) with an $\mathrm{Xbal}$ linker (5'-CTAGTCTAG ACTAG-3'; New England Biolabs) bearing nonsense codons in all three reading frames. This deletion prolongs the lifetime of the encoded message $\sim 10$-fold. Plasmid pSV-BFB was constructed in two steps. An MluI site was first introduced into plasmid pSVB10 (Shyu et al. 1989) by oligonucleotide-directed mutagenesis of the third and fourth $\beta$-globin codons (CATCTGTCC $\rightarrow$ CACGCGTCCl to generate plasmid pSVB101. Plasmid pSV-BFB was then created by replacing a 1.14-kb MluI-BglII fragment of pSVB101 with a $2.46-\mathrm{kb}$ Mlul-Bglll fragment of pF106 (Shyu et al. 1989).

Plasmid pBBB + ARE3 was generated from pBBB + ARE (Shyu et al. 1989) by oligonucleotide-directed mutagenesis of the human c-fos ARE present in the 3 -untranslated region of the hybrid $\mathrm{BBB}+\mathrm{ARE}$ gene. To construct pBBB36, an $\mathrm{XbaI}$ linker $\left(5^{\prime}\right.$-CTAGTCTAGACTAG- $\left.3^{\prime}\right)$ with nonsense codons in all three reading frames was inserted into the $\mathrm{NcO}$ site (filled-in) of $\mathrm{pBBB}$ located within the second $\mathrm{BBB}$ exon.

Plasmid templates for in vitro synthesis of complementary RNA probes were prepared as follows. Plasmid pT7a181 was generated by inserting a $0.27-\mathrm{kb}$ Aval-HindIII fragment of plasmid pSV $\alpha 1$ between the $A v a I$ and HindIII sites of pT7/T3 $\alpha-19$ (BRL). Plasmid pT7BBF was constructed in two steps. First, a $0.80-\mathrm{kb}$ BamHI-AccI (filled-in) fragment of pBBF (Shyu et al. 1989 ) that spanned the $\beta$-globin/c-fos junction was inserted between the BamHI and HincIl sites of pT $7 / T 3 \alpha-19$. Deletion of a $0.55-\mathrm{kb}$ BamHI-ApaI fragment from the resulting plasmid /ends made blunt with T4 DNA polymerase before ligation) generated pT7BBF and recreated the BamHI site. Plasmids pSP6BFB, pT7BG, and pSP6 133 have been described previously (Shyu et al. 19891.

\section{Acknowledgments}

We are especially grateful to Khandan Baradaran and Chyi-Ying Chen for help with plasmid constructions. This research was supported by U.S. Public Health Service grant CA4385 (to M.E.G.) and grants GM42720 and GM35769 (to I.G.B.). A.-B.S. was supported by a postdoctoral fellowship from the American Cancer Society, Massachusetts Division. M.E.G. was the recip- ient of American Cancer Society Junior Faculty Research Award JFRA-179 and was a Searle Scholar supported by the Chicago Community Trust.

The publication costs of this article were defrayed in part by payment of page charges. This article must therefore be hereby marked "advertisement" in accordance with 18 USC section 1734 solely to indicate this fact.

\section{References}

Almendral, J.M., D. Sommer, H. MacDonald-Bravo, J. Burckhardt, J. Perera, and R. Bravo. 1988. Complexity of the early genetic response to growth factors in mouse fibroblasts. Mol. Cell. Biol. 8: 2140-2148.

Baer, B. and R.D. Kornberg. 1980. Repeating structure of cytoplasmic poly(A) ribonucleoprotein. Proc. Nat1. Acad. Sci. 77: 1890-1892.

Bartel, D.P., M. Sheng, L.F. Lau, and M.E. Greenberg. 1989. Growth factors and membrane depolarization activate distinct programs of early response gene expression: Dissociation of fos and jun induction. Genes \& Dev. 3: 304-313.

Belasco, J.G. and C.F. Higgins. 1988. Mechanism of mRNA decay in bacteria: A perspective. Gene 72: 15-23.

Bernstein, P. and J. Ross. 1989. Poly(A), poly(A) binding protein, and the regulation of mRNA stability. Trends Biochem. Sci. 14: 373-377.

Bernstein, P., S.W. Peltz, and J. Ross. 1989. The poly(A)-poly(A)binding protein complex is a major determinant of mRNA stability in vitro. Mol. Cell. Biol. 9: 659-670.

Brawerman, G. 1981. The role of the poly(A) sequence in mammalian messenger RNA. CRC Crit. Rev. Biochem. 10: 1-38.

1987. Determinants of messenger RNA stability. Cell 48: $5-6$

Brewer, G. and J. Ross. 1988. Poly (A) shortening and degradation of the $3^{\prime} \mathrm{A}+\mathrm{U}$-rich sequences of human $\mathrm{c}-\mathrm{myc}$ mRNA in a cell-free system. Mol. Cell. Biol. 8: 1697-1708.

Caput, D., B. Beutler, K. Hartog, R. Thayer, S. Brown-Shimer, and A. Cerami. 1986. Identification of a common nucleotide sequence in the $3^{\prime}$-untranslated region of mRNA molecules specifying inflammatory mediators. Proc. Natl. Acad. Sci. 83: $1670-1674$.

Curran, T. and B.R. Franza. 1988. Fos and Jun: The AP-I connection. Cell 55: 395-397.

Drummond, D.R., J. Armstrong, and A. Colman. 1985. The effect of capping and polyadenylation on the stability, movement and translation of synthetic messenger RNAs in Xenopus oocytes. Nucleic Acids Res. 13: 7375-7394.

Feinberg, A.P. and B. Vogelstein. 1983. A technique for radiolabeling DNA restriction endonuclease fragments to high specific activity. Anal. Biochem. 132: 6-13.

Fort, P., J. Rech, A. Vie, M. Piechaczyk, A. Bonnieu, P. Jeanteur, and J.-M. Blanchard. 1987. Regulation of c-fos gene expression in hamster fibroblasts: Initiation and elongation of transcription and mRNA degradation. Nucleic Acids Res. 15: 5657-5667.

Galili, G., E.E. Kawata, L.D. Smith, and B.A. Larkins. 1988. Role of the 3'-poly(A) sequence in translational regulation of $\mathrm{mR}$ NAs in Xenopus laevis oocytes. I. Biol. Chem. 263: 5764 5770 .

Greenberg, M.E. and E.B. Ziff. 1984. Stimulation of 3T3 cells induces transcription of the c-fos proto-oncogene. Nature 311: 433-438.

Jackson, R.J. and N. Standart. 1990. Do the poly(A) tail and 3' untranslated region control mRNA translation? Cell 62: 1524. 
Jones, T.R. and M.D. Cole. 1987. Rapid cytoplasmic turnover of c-myc mRNA: Requirement of the $3^{\prime}$ untranslated sequences. Mol. Cell. Biol. 7: 4513-4521.

Kabnick, K.S. and D.E. Housman. 1988. Determinants that contribute to cytoplasmic stability of human c-fos and $\beta$-globin mRNAs are located at several sites in each mRNA. Mol. Cell. Biol. 8: 3244-3250.

Kates, J. 1970. Transcription of the vaccinia virus genome and the occurrence of polyriboadenylic acid sequences in messenger RNA. Cold Spring Harbor Symp. Quant. Biol. 38: $743-752$.

Kinniburgh, A.J., L.E. Maquat, T. Schedl, E. Rachmilewitz, and J. Ross. 1982. mRNA-deficient $\beta^{\circ}$-thalassemia results from a single nucleotide deletion. Nucleic Acids Res. 10: 54215427.

Kleene, K.C., R.J. Distel, and N.B. Hecht. 1984. Translational regulation and deadenylation of a protamine mRNA during spermatogenesis in the mouse. Dev. Biol. 105: 71-79.

Krowczynska, A., B. Yenofsky, and G. Brawerman. 1985. Regulation of messenger RNA stability in mouse erythroleukemia cells. J. Mol. Biol. 181: 231-239.

Lathe, R., M.P. Kieny, S. Skory, and J.P. Lecocq. 1984. Linkertailing: Unphosphorylated linker oligonucleotides for joining DNA termini. DNA 3: 173-182.

Lau, L.F. and D. Nathans. 1985. Identification of a set of genes expressed during the $\mathrm{G} 0 / \mathrm{Gl}$ transition of cultured mouse cells. EMBO /. 4: 3145-3151.

Levine, B.J., N. Chodchoy, W.F. Marzluff, and A.I. Skoultchi. 1987. Coupling of replication-type histone mRNA levels to DNA synthesis requires the stem-loop structure at the 3 ' end of the mRNA. Proc. Natl. Acad. Sci. 84: 6189-6193.

Lim, L. and E.S. Canellakis. 1970. Adenine-rich polymer associated with rabbit reticulocyte messenger RNA. Nature 227: 710-712

Lim, R.W., B.C. Varnum, and H.R. Herschman. 1987. Cloning of tetradecanoyl phorbol ester-induced "primary response" sequences and their expression in density-arrested Swiss 3T3 cells and a TPA non-proliferative variant. Oncogene 1: 263270.

Marbaix, G., G. Huez, A. Burny, Y. Cleuter, E. Hubert, M. Leclercq, H. Chantrenne, H. Soreq, U. Nudel, and U.Z. Littauer. 1975. Absence of polyadenylate segment in globin messenger RNA accelerates its degradation in Xenopus oocytes. Proc. Natl. Acad. Sci. 72: 3065-3067.

Meijlink, F., T. Curran, A.D. Miller, and I.M. Verma. 1985. Removal of a 67-base-pair sequence in the noncoding region of proto-oncogene fos converts it to a transforming gene. Proc. Natl. Acad. Sci. 82: 4987-4991.

Miller, A.D., T. Curran, and I.M. Verma. 1984. c-fos protein can induce cellular transformation: A novel mechanism of activation of a cellular oncogene. Cell 36: $51-60$.

Rahmsdorf, H.J., A. Schonthal, P. Angel, M. Litfin, U. Ruther, and P. Herrlich. 1987. Posttranscriptional regulation of c-fos mRNA expression. Nucleic Acids Res. 15: 1643-1660.

Rivera, V.M., M. Sheng, and M.E. Greenberg. 1990. The inner core of the serum response element mediates both the rapid induction and subsequent repression of c-fos transcription following serum stimulation. Genes \& Dev. 4: 255-268.

Sachs, A. and R.W. Davis. 1989. The poly(A) binding protein is required for poly $(\mathrm{A})$ shortening and $60 \mathrm{~S}$ ribosomal subunitdependent translation initiation. Cell 58: 857-867.

Sachs, A., R.W. Davis, and R.D. Kornberg. 1987. A single domain of yeast poly(A)-binding protein is necessary and sufficient for RNA binding and cell viability. Mol. Cell. Biol. 7: 3268-3276.

Sehgal, P.B., H. Soreq, and I. Tamm. 1978. Does 3'-terminal
poly(A) stabilize human fibroblast interferon mRNA in oocytes of Xenopus laevis? Proc. Natl. Acad. Sci. 75: 50305033.

Shapiro, R.A., D. Herrick, R.E. Manrow, D. Blinder, and A. Jacobson. 1988. Determinants of mRNA stability in Dictyostelium discoideum amoeba: Differences in poly $(\mathbf{A})$ tail length, ribosome loading, and mRNA size cannot account for the heterogeneity of mRNA decay rates. Mol. Cell. Biol. 8: 1957-1969.

Shaw, G. and R. Kamen. 1986. A conserved AU sequence from the 3 ' untranslated region of GM-CSF mRNA mediates selective mRNA degradation. Cell 46: 659-667.

Sheng, M. and M.E. Greenberg. 1990. The regulation and function of $\mathrm{c}$-fos and other immediate early genes in the nervous system. Neuron 4: 477-485.

Shyu, A.-B., M.E. Greenberg, and J.G. Belasco. 1989. The c-fos transcript is targeted for rapid decay by two distinct mRNA degradation pathways. Genes $\Leftrightarrow$ Dev. 3: 60-72.

Swartout, S.G. and A.J. Kinniburgh. 1989. c-myc RNA degradation in growing and differentiating cells: Possible alternate pathways. Mol. Cell. Biol. 9: 288-295.

Treisman, R. 1985. Transient accumulation of c-fos RNA following serum stimulation requires a conserved $5^{\prime}$ element and c-fos 3' sequence. Cell 42: 889-902.

Whittemore, L.-A. and T. Maniatis. 1990. Postinduction turnoff of beta-interferon gene expression. Mol. Cell. Biol. 64: 13291337.

Wilson, T. and R. Treisman. 1988. Removal of poly(A) and consequent degradation of c-fos mRNA facilitated by $3^{\prime}$ AU-rich sequences. Nature 336: 396-399. 


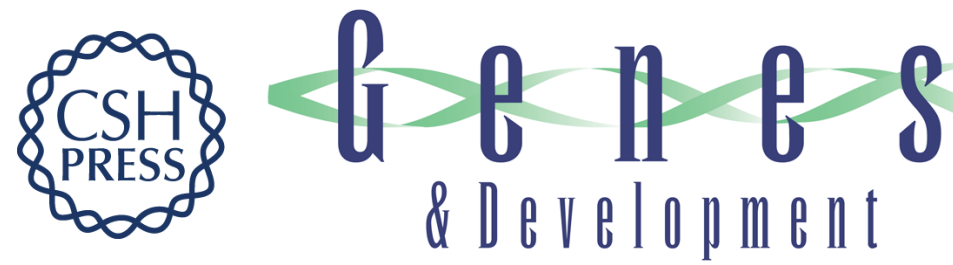

\section{Two distinct destabilizing elements in the c-fos message trigger deadenylation as a first step in rapid mRNA decay.}

A B Shyu, J G Belasco and M E Greenberg

Genes Dev. 1991, 5:

Access the most recent version at doi:10.1101/gad.5.2.221

References This article cites 44 articles, 18 of which can be accessed free at:

http://genesdev.cshlp.org/content/5/2/221.full.html\#ref-list-1

License

Email Alerting

Service

Receive free email alerts when new articles cite this article - sign up in the box at the top right corner of the article or click here.

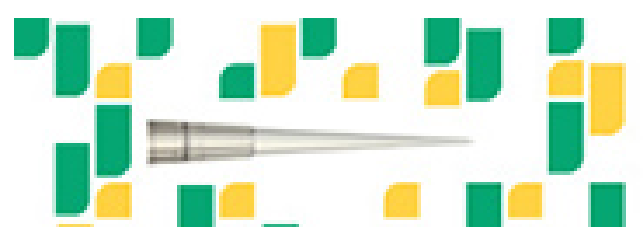

Focused on your science. 